\title{
Ductal carcinoma in situ of the breast: the importance of morphologic and molecular interactions.
}

\author{
Stacey K. Mardekian \\ Alessandro Bombonati \\ Juan P. Palazzo
}

Follow this and additional works at: https://jdc.jefferson.edu/pacbfp

\section{Let us know how access to this document benefits you}

\section{Recommended Citation \\ Mardekian, Stacey K.; Bombonati, Alessandro; and Palazzo, Juan P., "Ductal carcinoma in situ of the breast: the importance of morphologic and molecular interactions." (2016). Department of Pathology, Anatomy, and Cell Biology Faculty Papers. Paper 183.}

https://jdc.jefferson.edu/pacbfp/183

This Article is brought to you for free and open access by the Jefferson Digital Commons. The Jefferson Digital Commons is a service of Thomas Jefferson University's Center for Teaching and Learning (CTL). The Commons is a showcase for Jefferson books and journals, peer-reviewed scholarly publications, unique historical collections from the University archives, and teaching tools. The Jefferson Digital Commons allows researchers and interested readers anywhere in the world to learn about and keep up to date with Jefferson scholarship. This article has been accepted for inclusion in Department of Pathology, Anatomy, and Cell Biology Faculty Papers by an authorized administrator of the Jefferson Digital Commons. For more information, please contact: JeffersonDigitalCommons@jefferson.edu. 
Title: Ductal carcinoma in situ of the breast: The importance of morphologic and molecular interactions

Authors: Stacey K Mardekian, MDa, Alessandro Bombonati, MD ${ }^{b}$, Juan P Palazzo, $\mathrm{MD}^{\mathrm{c}}$

Affiliations:

a Department of Pathology, Thomas Jefferson University, Philadelphia, PA, USA Email: Stacey.Mardekian@jefferson.edu

${ }^{\mathrm{b}}$ Department of Pathology; Albert Einstein Medical Center; Philadelphia, PA, USA Email: BombonaA@einstein.edu

${ }^{c}$ Department of Pathology, Thomas Jefferson University, Philadelphia, PA, USA Email: Juan.Palazzo@jefferson.edu

Corresponding Author:

Juan P. Palazzo

132 S. $10^{\text {th }} \mathrm{St}$

285 Main Building

Philadelphia, PA 19107

Phone: 215-955-4403

Email: Juan.Palazzo@jefferson.edu

Summary:

Ductal carcinoma in situ (DCIS) of the breast is a lesion characterized by significant heterogeneity, in terms of morphology, immunohistochemical staining, molecular signatures and clinical expression. For some patients, surgical excision provides adequate treatment, but a subset of patients will experience recurrence of DCIS or progression to invasive ductal 
carcinoma (IDC). Recent years have seen extensive research aimed at identifying the molecular events that characterize the transition from normal epithelium to DCIS and IDC. Tumor epithelial cells, myoepithelial cells and stromal cells undergo alterations in gene expression, which are most important in the early stages of breast carcinogenesis. Epigenetic modifications, such as DNA methylation, together with microRNA (miRNA) alterations, play a major role in these genetic events. Additionally, tumor proliferation and invasion is facilitated by the lesional microenvironment, which includes stromal fibroblasts and macrophages that secrete growth factors and angiogenesis-promoting substances. Characterization of DCIS on a molecular level may better account for the heterogeneity of these lesions and how this manifests as differences in patient outcome and response to therapy. Molecular assays originally developed for assessing likelihood of recurrence in IDC are recently being applied to DCIS, with promising results. In the future, the classification of DCIS will likely incorporate molecular findings along with histologic and immunohistochemical features, allowing for personalized prognostic information and therapeutic options for patients with DCIS. This review summarizes current data regarding the molecular characterization of DCIS and discusses the potential clinical relevance.

Key words: ductal carcinoma in situ; invasive ductal carcinoma; biomarkers; gene expression; myoepithelial cells; tumor microenvironment

Running head: Morphologic and molecular interactions in DCIS

Conflicts of interest:

None

Acknowledgements:

We would like to thank Paul Schiffmacher for his contribution to the design of Figure 1. 


\section{Introduction}

DCIS accounts for approximately $20 \%$ of all newly diagnosed breast cancer cases in the United States [1]. DCIS is defined as a neoplastic proliferation of epithelial cells with varying degrees of cytological atypia that are confined to the mammary ductal-lobular system. DCIS itself does not result in mortality, and breast cancer-specific mortality among women with DCIS is extremely low with 1.0-2.6\% dying from invasive breast cancer (IBC) 8-10 years after a diagnosis of DCIS [2]. Due to the non-invasive nature and overall favorable prognosis of DCIS, a 2009 National Institutes of Health State-of-the-Science Conference issued a statement which advocated elimination of the term "carcinoma" in the name of this lesion [3]. Since DCIS is a non-obligate precursor of invasive ductal carcinoma (IDC), an invasive component is found in a subset of women who develop DCIS recurrence [4]. Furthermore, experimental data has shown that carcinoma precursor cells exist in DCIS lesions, suggesting that the aggressive phenotype of breast cancer is predetermined early at the pre-malignant stage [5].

Significant advances have been made in the diagnosis and therapy of patients with ductal carcinoma in situ of the breast (DCIS). Early detection has led to an increase in DCIS cases seen by pathologists in their daily practice. While a majority of women are treated by surgical excision followed by radiation therapy and anti-hormonal medications, a number of series have also demonstrated the natural course of untreated DCIS $[4,6,7]$. While data demonstrates that there could be a population of patients who can safely undergo observation after a breastconserving surgery, many physicians and patients are reticent to undergo this course of treatment for fear of recurrence $[7,8]$. Fifty percent of DCIS recurrence actually presents as an invasive cancer; therefore, consequences could be significant [9]. Thus, it is likely that a significant number of patients are being overtreated. 
However, considering potential long-term side effects of radiation therapy, it seems prudent to identify a population of patients to observe without further therapy. Studies have shown that certain clinical and pathological features of DCIS may be prognostic of local and/or invasive recurrence following surgical excision $[6,7,10]$. For instance, many studies have suggested that the tumor size is a strong predictor of local recurrence $[7,11]$. Nuclear grade and margin status are other factors that appear to influence recurrence in DCIS, though certainly there are studies that refute these findings [12]. Two major clinical tools that aid in risk stratification and treatment planning--the University of Southern California/Van Nuys Prognostic Index and the DCIS nomogram introduced by Rudloff et al.--utilize both clinical and pathologic factors such as tumor size, necrosis, and margin status $[10,13,14]$. While our current ability to accurately predict recurrence on the basis of this parameters is limited, clearly the paradigm that "one approach fits all" in patients with DCIS is shifting. The current understanding is that DCIS is not one disease but a heterogeneous group of cancers with distinct morphologic, immunohistochemical, and biological features.

Recent research efforts have focused on classifying patients who may be managed conservatively and those who are at higher risk for disease progression and may require adjuvant therapy. The ideal classification scheme would be clinically useful and easy to adapt with the ability to stratify patients into prognostic groups. While not consistently identified in all studies, certain histopathological parameters such as lesion size, margin status, architectural pattern, nuclear grade, presence of comedo necrosis, and expression of various immunohistochemical markers have been variably shown to affect the risk of recurrence in premalignant breast lesions [12]. Improved molecular characterization of DCIS will offer additional, perhaps more definitive, prognostic information and may provide the opportunity for personalized therapeutic options for patients with DCIS. 


\section{DCIS carcinogenesis}

There is a continuum of non-obligate precursor lesions to IDC, consisting of flat epithelial atypia (FEA), atypical ductal hyperplasia (ADH), and DCIS. Moreover, low-grade and high-grade DCIS likely arise from two distinct evolutionary pathways. FEA is genetically related to ADH and is likely a precursor to $\mathrm{ADH}$, which, in turn, is the precursor to low-grade DCIS $[4,15]$.

The pathophysiology of the malignant transformation from DCIS to IDC has been studied at the molecular level. Comparative genomic hybridization studies of synchronous and metachronous DCIS and IDC lesions have revealed a near identical pattern of genomic alterations, correlating with tumor grade, supporting a molecular continuum between DCIS and IDC. Specifically, lowgrade lesions harbor frequent loss of $16 q$ and $17 p$, while high grade lesions have complex genomic alterations including $13 q$ loss and high level amplifications of $17 q 12$ and 11q13 [16]. The use of gene expression microarray technology has further refined our knowledge of DCIS as a heterogeneous disease and has provided a new classification based on molecular signatures. Ma et al discovered that unique gene expression signatures are associated with different tumor grades, irrespective of tumor stage [17]. ADH, low-grade DCIS, and low-grade IDC share a near identical gene expression profile consisting of genes associated with the estrogen receptor phenotype, whereas both high-grade DCIS and high-grade IDC possess a unique gene expression profile consisting of genes associated with mitotic activity and cell cycle processes. Additionally, these authors identified a subset of genes with quantitative expression levels that correlate with advanced tumor grade and with the transition from DCIS to IDC. This suggests that the transcriptional program that drives cancer cells to an advanced tumor grade may also confer invasiveness. Specifically, the gene ribonucleotide reductase M2 (RRM2) may play a dual role in both supporting rapid cell proliferation and promoting invasive growth behavior [17]. In the transition to invasive disease, low-grade DCIS lesions give rise to welldifferentiated IDC after a long latency period, and high-grade DCIS lesions give rise to poorly 
differentiated IDC after a relatively shorter time period [18]. Using a supervised classification, Hannemann et al. identified a gene expression classifier of 35 genes, which differed between DCIS and IDC and a panel of 43 genes which could further separate between well- and poorly differentiated DCIS samples [19]. These findings were confirmed by Castro et al. who showed that the tumor cells with the most divergent molecular features were from the pure DCIS cases, providing further evidence that molecular changes in cells occur before morphological alterations during the progression of IDC [20]. More recently, Lee and colleagues described a 74-gene profile which was able to correctly categorize $97 \%$ of all DCIS and 95\% of all IBCs [21]. In addition, the performance of this signature was evaluated in cohorts from three similar independent studies [21-24]. The discovery of distinct molecular subtypes in DCIS has further validated these lesions as precursors of the different subtypes of invasive breast carcinomas. Surprisingly, the tumor epithelial cells do not demonstrate significant qualitative gene expression changes in the transition from in situ to invasive carcinoma. Instead the most dramatic transcriptome changes take place prior to local invasion during the transition from normal epithelium to DCIS $[17,25]$. In summary, the molecular data have shown that qualitatively, the epithelial components of synchronous DCIS and IDC are remarkably similar.

\section{Molecular pathology of DCIS}

An important question regarding the molecular analysis of DCIS is to determine whether the molecular subtypes identified in invasive cancers have the same significance in patients with DCIS. All of the major molecular subtypes present in IBC (luminal A, luminal B, HER2+, and basal-like) are seen in DCIS, although at different frequencies. Specifically, the frequency of the luminal B and HER2+ phenotypes is significantly higher in DCIS than in IDC, and the frequency of the luminal A phenotype is significantly higher in IDC than in DCIS [26]. Additionally, the molecular subtypes correlate with grade. Low-grade DCIS is associated with ER positivity and luminal phenotypes, while high-grade DCIS is associated with negative ER, positive HER2, and 
the basal-like phenotype [26]. The basal-like subtype seems to be associated with a higher risk of local recurrence than are luminal subtypes, and may even have stronger and more consistent associations than some of the conventional histopathological factors [10,27].

One of the interesting aspects of DCIS is that the expression of HER2 has been linked to initiation and progression of breast cancer. Therefore, its frequent overexpression--commonly seen in high grade DCIS with or without necrosis--suggests that HER2 plays a role in tumor initiation and progression [28]. HER2 is a member of the type I receptor tyrosine kinase family, which consists of four closely related family members: HER2, EGFR (HER1), HER3, and HER4. The major role of HER2 is to serve as a co-receptor in the dimerization and activation of other ERbB receptors. The amplification of the gene HER2 results in a 50 to 100 fold increase in the number of surface HER2 receptors on cancer cells compared to normal mammary cells [29]. One of the possible mechanisms by which HER2 mediates breast carcinogenesis is through its action on breast stem cells. HER2 promotes carcinogenesis by maintaining and increasing cancer stem cells [30]. Interestingly, stem cells that overexpress HER2 are more sensitive to trastuzumab and lapatinib [31]. In some studies, the expression of HER2/neu in DCIS has been linked to recurrence following surgical excision without radiation therapy $[7,32]$. In our series of DCIS patients treated with surgery alone, the expression of HER2 was associated with higher recurrence rate and increased incidence of invasive recurrences in patients followed after many years [7]. However, HER2 expression as an independent risk factor for recurrence remains controversial. The expression of HER2 in DCIS and the existence of HER2-expressing stem cells raise interesting issues regarding the possibility of using anti-HER2 compounds for targeted therapy. These options are presently being investigated in clinical trials with the use of anti-HER2 medications and anti-HER2 vaccines [33].

In addition to HER2 and steroid receptors, additional molecular markers may facilitate improved 
categorization of DCIS [34]. Other potential biomarkers that have been evaluated include proliferation markers, cell cycle regulation and apoptotic markers, angiogenesis-related proteins, cell adhesion molecules, epidermal growth factor receptor family receptors, extracellular matrix proteins, and cyclooxygenase type-2 (COX-2) [35]. DCIS lesions with a low likelihood of recurrence after surgical excision and of progression to invasive cancer are usually hormone receptor positive, with normal levels of p53, lack of HER2 amplification, and low levels of Ki67 [18]. DCIS lesions with a high potential for recurrence after surgical excision and for progression to invasive disease over a relatively short period of time are associated with HER2 amplification, hormone negative receptor status, presence of TP53 mutation, and increased levels of Ki67 [18]. COX-2 has also been shown to be significantly up-regulated in DCIS with higher cell proliferation rates, nuclear grade, ER negativity, and HER-2/neu positivity [18]. Berman et al showed that DCIS showing overexpression of Ki67 and p16 by immunohistochemistry are more likely to recur compared to tumors with low levels of expression [12].

\section{Clinically validated prognostic markers for DCIS}

The primary clinical dilemma in the management of DCIS patients relies on the fact that traditional clinicopathologic features do not accurately predict in which patients the disease will recur. Great advances have been made in the use of molecular profiling of invasive cancer for risk assessment; however, its implementation in clinical practice for the study of DCIS is lagging behind. Recently, a modified form of the Oncotype DX® recurrence score for IBC has been developed for DCIS. This RT-PCR based assay is based on 12 genes from the Oncotype DX® Invasive Recurrence Score. The algorithm uses 7 cancer-related genes (Ki67, STK15, Survivin, CCNB1, MYBL2, PR and GSTM1) and 5 reference genes, to generate a score that quantifies the likelihood of cumulative local recurrence (DCIS or invasive carcinoma) at 10 years and predicts the risk of an invasive carcinoma local event at 10 years. The assay was initially evaluated in a prospective study that included 327 of the 670 patients enrolled in the Eastern 
Cooperative Oncology Group (ECOG) 5149 clinical trial, who underwent surgery without radiation. Approximately $30 \%$ of patients received tamoxifen. In the ECOG trial, patients were divided in 2 cohorts: cohort 1 had low- or intermediate-grade DCIS measuring $\leq 2.5 \mathrm{~cm}$; cohort 2 had high-grade DCIS measuring $\leq 1 \mathrm{~cm}$ with surgical margins $\geq 3 \mathrm{~mm}$. Median age at surgery was 60 years, and median tumor size was $6 \mathrm{~mm}$. This study showed a 10-year risk for three pre-specified DCIS risk groups of $10.6 \%$ (low), $26.7 \%$ (intermediate), and $25.9 \%$ (high). The differences in the risks of developing local recurrence and invasive local recurrence between patients with a lower DCIS Score and a higher DCIS Score were statistically significant and clinically meaningful. The DCIS Score result was the strongest predictor of the risk of local recurrence in the validation study. Among standard clinical and pathologic characteristics, only tumor size and postmenopausal status were significant predictors of local recurrence, whereas tumor grade and comedo necrosis were not (when the DCIS Score result was excluded from the analysis). The power of this study was somewhat limited by the lack of tamoxifen randomization in the ECOG trial, by the fact that gene selection and algorithm development were based partly on studies of invasive carcinomas, and by the inclusion of only relatively few patients with hormone receptors negative or HER2 positive DCIS [36].

A recent publication has demonstrated the value of Oncotype DX® DCIS score in a larger population-based cohort of 718 patients diagnosed with DCIS treated with breast-conserving surgery alone with validation of treatment and outcomes. Tissue blocks from 571 patients with negative margins were available for the study. At a median follow-up of 9.6 years, 100 cases of local recurrence were identified (57 invasive and 44 DCIS). Using the Oncotype DX® DCIS score retrospectively, the 10 -year risk of local recurrence was estimated at $12.7 \%$ for low-risk patients, $27.8 \%$ for intermediate-risk patients, and 33\% for high-risk patients. The score also predicted invasive recurrence and DCIS recurrence. The outcomes of this cohort were remarkably similar to the ECOG's study one. Limitations of the study included the fact that 
patients were not randomized, and the use of and compliance with tamoxifen treatment was limited [37]. Additionally, it is often difficult to differentiate between true recurrence and the appearance of a second primary carcinoma, which is a limitation to all studies involving breast cancer recurrence. Lastly, for women with low DCIS scores, the 10-year local recurrence rates approached $11 \%$ and $13 \%$ in these two major studies, rates which many clinicians deem not low enough to justify omission of radiation therapy. Therefore, this assay has not yet been universally implemented into clinical practice.

In light of the numerous biomarkers that are being investigated for their role in the pathogenesis of DCIS, it is important to remember the current recommendations for routine practice of pathology. ER is currently the only biomarker validated for routine clinical practice in DCIS, and the National Comprehensive Cancer Network practice guidelines include determination of ER status as part of the work-up of DCIS [2,38]. The majority $(75-80 \%)$ of DCIS cases are ER positive. This information is used to determine if a patient with DCIS will benefit from hormonal therapy. However, the Update Committee of the American Society of Clinical Oncology concluded that current data are insufficient to make a general recommendation for the use of ER status of DCIS to make decisions about tamoxifen treatment [39]. Furthermore, even though PR is often ordered in conjunction with ER, there is almost no data on the association of PR status and DCIS. Thus, a surgical pathologist should decide which markers to routinely perform upon consultation with the clinicians who will use this information.

In our practice, all cases of DCIS are tested for ER, PR, HER2 and Ki67 expression. While we are not advocating this as universal routine practice, we have observed that the expression of these markers can sometimes affect the treatment decisions of clinicians at our institution. Additionally, building a cohort of cases tested with this panel of markers will allow us to observe their patterns of expression and investigate how they may underlie biologic differences in DCIS 
lesions. Other biomarkers currently under investigation could potentially become part of routine ordering practice, should they demonstrate prognostic or therapeutic significance. Available molecular tests such as Oncotype $D X ®$ also have the potential to become useful for predicting outcome in certain patients, especially since comprehensive histopathologic analysis alone does not seem to be sufficient for evaluating recurrence risk. Certainly, the impact of the costs of molecular tests and their application to a wide spectrum of patients with DCIS need to be analyzed in order to maximize their effect on patient management. We envision a future where we can better stratify patients with DCIS using a combination of pathologic examination, immunohistochemical studies and molecular analysis.

\section{The transition from DCIS to invasive carcinoma}

One of the areas of interest in recent years in patients with DCIS has been the study of the cells' ability to invade, grow in the stroma, and acquire metastatic potential. All of these steps are crucial, since preventing the tumor cells from becoming invasive is one of the major goals in the treatment of DCIS. Myoepithelial cells, stromal cells, vascular supply, and cell-mediated immune mechanisms all play a role in the transition from DCIS to invasive cancer [Figure 1]. The key morphological distinction between DCIS and IDC is the preservation of the myoepithelial cell layer in DCIS; the lack of myoepithelial cells is considered one of the hallmarks of invasive carcinoma [40].

Myoepithelial cells are known to be biologically different in normal and neoplastic breast tissue. In DCIS, they function as tumor suppressors, inhibitors of the invasive properties of the epithelial cells and angiogenesis [40,41]. Loss of myoepithelial cells is routinely tested for with immunohistochemical markers such as p63, calponin, smooth muscle actin, CD10, cytokeratin (CK) 5/6, S-100 protein, and smooth muscle myosin heavy chain (SMMHC). Heterogeneity in the staining patterns can be seen with these markers, which has prompted pathologists to use 
more than one marker to exclude invasive carcinoma. Altered expression of these markers has been observed in the myoepithelial cells of DCIS lesions. Hilson et al. were the first to report reduced staining intensity of DCIS-associated myoepithelial cells with various myoepithelial cell markers, lending support to the idea that these myoepithelial cells are phenotypically abnormal [42]. These authors also found that SMMHC, CD10 and CK 5/6 have lower sensitivity for DCISassociated myoepithelial cells than do SMA, p63 and calponin [42]. At our institution, we used two of the most common markers, p63 and calponin, in a large series of DCIS cases which revealed heterogeneous expression of these myoepithelial markers [Figure 2]. In this retrospective experience, we saw a marked decreased in the expression of these markers in high grade DCIS with necrosis, pleomorphic lobular carcinoma in situ, and in DCIS with associated invasive cancers. In contrast to these tumors, classic lobular carcinoma in situ and low grade DCIS showed strong and continuous expression of these two markers. This study showed significant heterogeneity according to the lesions and to the markers applied and emphasizes the importance of using more than one antibody.

The reasons for the immunohistochemical heterogeneity and for the decreased and absence of myoepithelial cells in the pre-invasive phase are not currently known. Two theories have been proposed regarding the mechanism by which myoepithelial cells are lost. One hypothesis suggests that genetic changes in tumor epithelial cells may eventually select for a clone with invasive properties that can escape from the duct, spread into the stroma, and subsequently expand [43]. However, countering this hypothesis are studies demonstrating that the tumor epithelial cells do not undergo significant gene expression changes in the transition from DCIS to IDC [17]. The second hypothesis suggests that phenotypic changes in DCIS myoepithelial cells, together with accumulation of stromal inflammatory cells and myofibroblasts, lead to breakdown of the ducts and release of the tumor epithelial cells into the surrounding stroma $[41,43]$. Many of the genes that are specific for normal myoepithelial cells, such as CTK14, 
CTK17, OXTR and EGFR, have been shown to be absent or dramatically downregulated in the myoepithelial cells of DCIS lesions [43]. Not only do the DCIS myoepithelial cells lack their normal tumor suppressive abilities, they may instead possess a reversed function, with the ability to promote tumor progression [44].

The surprising lack of genomic evolution between DCIS and IDC has led researchers to focus on the potential role of the stromal microenvironment in mediating the transition to invasion. It is known that the tumor-associated stromal cells secrete factors such as vascular endothelial growth factor, IL-6, and IL-8, which promote angiogenesis and breast cancer progression [45]. In contrast to the tumor epithelial cells, the tumor-associated stromal cells demonstrate distinct gene expression changes during the transition from in situ to invasive carcinoma. Ma et al. showed that in stromal cells, the transition to invasive growth is accompanied by increased expression of several matrix metalloproteases including MMP2, MMP11, and MMP14 [46]. Interestingly, the vast majority (>90\%) of the stromal gene expression changes observed between normal tissue and IDC occur early at the normal to DCIS transition [47]. Allinen et al. conducted an elegant comprehensive molecular characterization of each cell type (epithelial, myoepithelial and various stromal cells) composing normal breast tissue, in situ and invasive carcinoma to better understand the role of these cells in breast carcinogenesis [48]. The authors found that extensive gene expression changes occur in all cell types during cancer progression, with the most consistent and dramatic changes affecting myoepithelial cells. Surprisingly, genetic clonal alterations were detected only in cancer epithelial cells [48]. These findings were confirmed by another independent study that showed no evidence of clonal somatic genetic alterations in cancer-associated fibroblasts from human breast and ovarian carcinomas [49]. The contribution of both the epithelial and stromal compartments to the clinically important scenario of progression from DCIS to IDC has been shown by another independent study [50]. 
Muggerud et al. identified a set of genes independent of grade, ER-status, and HER2-status which allowed them to classify a subgroup of high-grade DCIS with a more active stroma and epithelial-mesenchymal transition (EMT) markers, molecularly similar to "more advanced" lesions such as invasive carcinoma [51]. Sharma et al also shed more light on the interaction between tumor cells and their surrounding stroma in model systems. These authors identified two distinct stromal signatures derived from a macrophage response and a fibroblastic response that were present in subsets of invasive and in-situ carcinomas [52]. Of interest, the macrophage response signature was associated with a more aggressive phenotype, higher grade DCIS and cases which were ER and PR negative, whereas the fibroblast signature was not associated with any clinicopathologic features in DCIS. This study suggests the role of the microenvironment as a potential "driver" of disease behavior [52]. Gene expression profiling has revealed that substantial changes occur during progression from DCIS to IBC in various cell types of the tumor stromal microenvironment; however, no clonal genetic aberrations have been detected in the myoepithelial cells and fibroblasts surrounding DCIS or IBC $[44,46]$.

Further support to the increasing evidence that changes in the cellular microenvironment contribute to tumorigenesis has been provided by $\mathrm{Hu}$ et al. The authors analyzed epithelial, myoepithelial cells, and fibroblasts from normal, in situ and invasive breast cancers, through methylation-specific digital karyotyping and found consistent differences in DNA methylation between normal and neoplastic breast tissue in each cell type analyzed [53]. Recently, Fleischer and colleagues also investigated methylation patterns during progression of breast cancer [54]. Genome wide DNA methylation analysis was carried out in a total of 285 fresh frozen tissue samples, including 46 normal breast tissue samples from healthy women, 22 pure DCIS, 31 mixed DCIS-IBC and 186 IBC. The authors concluded that most of the aberrations in the epigenetic profile were observed already in the pre-invasive DCIS stage, while the changes between DCIS and IBC were comparably modest [54]. 
6. The role of methylation analysis in DCIS

DNA methylation is an epigenetic modification of the human genome that is implicated in cancer. The altered DNA methylation patterns found in breast cancer cells include both hypomethylation and hypermethylation of specific gene regions. In particular, promoter hypermethylation has been implicated as an early event in breast carcinogenesis [55]. The role of methylation in DCIS and breast cancer progression has been investigated in numerous studies that used different methodologies, analyzed different gene sets, and examined different promoter regions [56,57].

In brief, methylation studies have showed that the number of methylated genes increased stepwise from normal breast to DCIS, whereas IDC did not differ from DCIS. These findings suggest that most of the dramatic epigenetic changes take place during the transition from normal epithelium to DCIS and that aberrant methylation may not contribute to the development of invasion but rather play an important role in early breast carcinogenesis [58-60]. Recent studies, some of which have used a genome-wide methylation screen approach have described a small set of genes such as APC, CACNA1A, CDH1, FOXC1, HOXA10, MGMT, SFPR1, TFAP2A, and TWIST1 that exhibit differences in either frequency or density of methylation between DCIS and invasive carcinoma [61-70]. In view of these findings, the analysis of such a gene panel through quantitative methylation might represent a tool for predicting disease progression. These data have been confirmed by Fleischer et al. who recently reported the DNA methylation profiles of a breast cancer progression series, including normal breast tissue, DCIS, invasive carcinoma, and mixed lesions [54]. In agreement with previous studies, most of the aberrations in the epigenetic profile were observed already in the pre-invasive stage. Of note the authors also described a signature comprising DNA methylation levels of $18 \mathrm{CpGs}$ that was prognostic for breast cancer patients with invasive tumors as well as for patients with DCIS and 
mixed lesions of DCIS and invasive breast carcinoma [54].

\section{MicroRNAs and DCIS}

MicroRNAs (miRNAs) are a class of small noncoding RNAs that control gene expression by targeting mRNAs and triggering either translation repression or RNA degradation [71]. miRNAs

have been shown to control several cellular processes, including metabolism, stem cell division, cell growth and differentiation and apoptosis [72-76]. The effect of miRNAs on mRNA expression and its contribution to the pathogenesis of many diseases, including mammary carcinogenesis has been elucidated in the last decade [77-83]. In a seminal study, lorio et al. demonstrated aberrant expression of miRNA, notably miR-125b, miR-145, miR-21, and miR155, in human breast cancer. Furthermore, the authors identified miRNAs whose expression was correlated with specific breast cancer biopathologic features, such as estrogen and progesterone receptor expression, tumor stage, vascular invasion, or proliferation index [84]. Hannafon et al. performed an integrated analysis of miRNA and mRNA expression in paired samples of histologically normal and pre-invasive breast cancer and found that major miRNA expression changes occur at the transition from normal to DCIS epithelium [85]. The authors further demonstrated, by modulating the expression of several miRNA, that the expression of their predicted target genes is affected. This work implicated the loss of the tumor suppressor miR-125b and the gain of the oncogenic miRNA miR-182 and miR-183 as major contributors to early breast cancer development [85]. Farazi et al. profiled miRNAs from normal breast tissues, DCIS, invasive breast carcinomas, and 6 cell lines by Solexa sequencing and showed that normal breast samples were separated from most noninvasive DCIS and invasive carcinomas by increased miR-21 (the most abundant miRNA in carcinomas) and multiple decreased miRNA families, with most miRNA changes apparent already in the noninvasive carcinomas [86]. In essence, the study confirmed prior findings and supported the view that most miRNA changes in IDC were already apparent in DCIS samples [86]. Similarly Volinia et al. created miRNA 
profiles for normal breast, DCIS, and invasive carcinomas to study the global changes of the miRNA repertoire along the transition defining breast carcinoma progression [87]. The miRNA profile established for the normal breast to DCIS transition was largely maintained in the in situ to IDC progression. Nevertheless, a nine-miRNA signature was identified that differentiated invasive from in situ carcinoma along with five miRNAs associated with time to metastasis and overall survival in IDC patients. Of clinical interest miR-210, which resulted to be regulated during breast cancer progression, was also a component of the two prognostic signatures [87].

\section{Next-generation sequencing}

Next-generation sequencing (NGS) technologies are revolutionizing our ability to characterize breast cancer at the genomic, transcriptomic and epigenetic levels. Unlike microarrays, that have been the technology of choice in most gene expression studies, NGS does not depend on prior sequence information; hence, the identification and quantification of gene expression is unbiased. Moreover NGS offers unprecedented depth of analysis of gene expression and is not affected by potential cross hybridization [88-91]. NGS has been already exploited in DCIS with the production of massive quantity of data, and this is just the beginning. Kaur et al. used next generation sequencing to identify the transcriptional fingerprint of three DCIS models at the whole genome level [92]. This remarkable study revealed significant increases in ALDH5A1 (enzyme of glutamate metabolism) expression in the DCIS models and demonstrated that two independent drugs (disulfiram and valproic acid) reduce net proliferation in DCIS by inhibiting ALDH5A1 activity [92]. In 2012 Banerij et al. performed whole-exome sequencing of 103 primary, treatment-naive breast carcinomas including 9 cases of DCIS. Although the results pertinent to the small subset of DCIS samples were not separately elucidated from the entire set of 103 cancers, the overall data set exhibited recurrent somatic mutations in 5 known genes-PIK3CA, TP53, AKT1, GATA3, and MAP3K1--and discovered new mutations in the CBFB transcription factor gene along with deletions of its partner RUNX1 [93]. 


\section{Conclusions}

With the increased frequency of breast cancers detected at the non-invasive stage, pathologists play an increasingly significant role. This role is not limited to the diagnosis (benign versus malignant) but to providing important information to guide treatment decisions in patients with $\mathrm{DCIS}$ and to determine the risk of recurrence and progression to invasive carcinoma. DCIS is a heterogeneous lesion which can demonstrate different morphological patterns and express combinations of immunohistochemical and molecular markers. A better understanding of the molecular events of the lesions preceding DCIS and of the DCIS itself will lead to better methods of prevention and treatment options for these patients. The biological changes in the various cellular components of DCIS, including the tumor epithelial cells, myoepithelial cells, and stromal cells, may hold the key to whether or not a given lesion will progress to invasive carcinoma.

The progression from in situ to invasive breast cancer is a very complex biological phenomenon. The cells in DCIS lesions that do progress to invasive cancer likely already harbor molecular alterations that render them prone to progress and become invasive. Therefore, defining DCIS on a molecular basis may be a more accurate way of accounting for the frequent heterogeneity of these lesions and how this manifests as differences in patient outcome and response to therapy. Testing DCIS lesions with molecular markers may better predict the risk of disease progression. Overcoming tumor heterogeneity will be a major challenge to identify the genes driving the progression of DCIS. In the future, the classification of DCIS will include histologic, immunohistochemical, and molecular markers, in order to tailor a patient's management to the biological nature and malignant potential of their individual DCIS lesions. Newer treatment modalities in clinical trials such as anti-HER2 compounds, vaccines, inhibitors of angiogenesis, and growth factors await the results to evaluate their implementation in clinical 
practice.

References:

[1] Siziopikou KP. Ductal carcinoma in situ of the breast: current concepts and future directions. Arch Pathol Lab Med 2013;137:462-6.

[2] Schnitt S, Allred C, Britton P, et al. Ductal carcinoma in situ. In: Lakhani SR, Ellis IO, Schnitt SJ, Tan PH, van de Vijver MJ, editors. WHO Classification of Tumours of the Breast, Lyon: IARC Press; 2012, p. 90-4.

[3] Allegra CJ, Aberle DR, Ganschow P, et al. National Institutes of Health State-of-the-Science Conference statement: Diagnosis and Management of Ductal Carcinoma In Situ September 2224, 2009. J Natl Cancer Inst 2010;102:161-9.

[4] Bombonati A, Sgroi DC. The molecular pathology of breast cancer progression. J Pathol $2011 ; 223: 307-17$.

[5] Espina V, Liotta LA. What is the malignant nature of human ductal carcinoma in situ? Nat Rev Cancer 2011;11:68-75.

[6] Silverstein MJ, Lagios MD. Choosing treatment for patients with ductal carcinoma in situ: fine tuning the University of Southern California/Van Nuys prognostic index. J Natl Cancer Inst Monogr 2010;41:193-6.

[7] Holmes P, Lloyd J, Chervoneva I, et al. Prognostic markers and long-term outcomes in ductal carcinoma in situ of the breast treated with excision alone. Cancer 2011;117:3650-7. [8] Wong JS, Kaelin CM, Troyan SL, et al. Prospective study of wide excision alone for ductal carcinoma in situ of the breast. J Clin Oncol 2006;24:1031-6.

[9] Virnig BA, Tuttle TM, Shamliyan T, Kane RL. Ductal carcinoma in situ of the breast: a systematic review of incidence, treatment and outcomes. J Natl Cancer Inst 2010;102(3):170-8. [10] Benson JR, Wishart GC. Predictors of recurrence for ductal carcinoma in situ after breastconserving surgery. Lancet Oncol 2013;14:e348-57. 
[11] Kantor O, Winchester DJ. Breast conserving therapy for DCIS—does size matter? J Surg Oncol 2014;110:75-81.

[12] Berman HK, Gauthier ML, Tisty TD. Premalignant breast neoplasia: a paradigm of interlesional and intralesional molecular heterogeneity and its biological and clinical ramifications. Cancer Prev Res 2010;3:579-87.

[13] Rudloff U, Jacks LM, Goldberg JI, et al. Nomogram for predicting the risk of local recurence after breast-conserving surgery for ductal carcinoma in situ. J Clin Oncol 2010;28:3762-9. [14] Yi M, Meric-Bernstam F, Kuerer HM, et al. Evaluation of a breast cancer nomogram for predicting risk of ipsilateral breast tumor recurrences in patients with ductal carcinoma in situ after local excision. J Clin Oncol 2012;30:600-7.

[15] Sgroi DC. Preinvasive breast cancer. Annu Rev Pathol 2010;5:193-221.

[16] Buerger $\mathrm{H}$, Otterbach F, Simon R, et al. Different genetic pathways in the evolution of invasive breast cancer are associated with distinct morphological subtypes. J Pathol 1999;189:521-6.

[17] Ma XJ, Salunga R, Tuggle JT, et al. Gene expression profiles of human breast cancer progression. Proc Natl Acad Sci USA 2003;100:5974-9.

[18] Tsikitis VL, Chung MA. Biology of ductal carcinoma in situ classification based on biologic potential. Am J Clin Oncol 2006;29:305-10.

[19] Hannemann J, Velds A, Halfwerk JB, Kreike B, Peterse JL, van de Vijver MJ. Classification of ductal carcinoma in situ by gene expression profiling. Breast Cancer Res 2006;8:R61. [20] Castro NP, Osorio CA, Torres C, et al. Evidence that molecular changes in cells occur before morphological alterations during the progression of breast ductal carcinoma. Breast Cancer Res 2008;10:R87.

[21] Lee S, Stewart S, Nagtegaal I, et al. Differentially expressed genes regulating the progression of ductal carcinoma in situ to invasive breast cancer. Cancer Res 2012;72:4574-86. [22] Schuetz CS, Bonin M, Clare SE, et al. Progression-specific genes identified by expression 
profiling of matched ductal carcinomas in situ and invasive breast tumors, combining laser capture microdissection and oligonucleotide microarray analysis. Cancer Res 2006;66:5278-86. [23] Knudsen ES, Ertel A, Davicioni E, Kline J, Schwartz GF, Witkiewicz AK. Progression of ductal carcinoma in situ to invasive breast cancer is associated with gene expression programs of EMT and myoepithelia. Breast Cancer Res Treat 2012;133:1009-24.

[24] Kretschmer C, Sterner-Kock A, Siedentopf F, Schoenegg W, Schlag PM, Kemmner W. Identification of early molecular markers for breast cancer. Mol Cancer 2011;10:15. [25] Porter D, Lahti-Domenici J, Keshaviah A, et al. Molecular markers in ductal carcinoma in situ of the breast. Mol Cancer Res 2003;1:362-75.

[26] Tamimi RM, Baer HJ, Marotti J, et al. Comparison of molecular phenotypes of ductal carcinoma in situ and invasive breast cancer. Breast Cancer Res 2008;10:R67.

[27] Livasy CA, Perou CM, Karaca G, et al. Identification of a basal-like subtype of breast ductal carcinoma in situ. Hum Pathol 2007;38:197-204.

[28] Roses RE, Paulson EC, Sharma A, et al. HER-2/neu overexpression as a predictor for the transition from in situ to invasive breast cancer. Cancer Epidemiol Biomarkers Prev 2009;18:1386-9.

[29] Wilson CA, Cajulis EE, Green JL, et al. HER-2 overexpression differentially alters transforming growth factor- $\beta$ responses in luminal versus mesenchymal human breast cancer cells. Breast Cancer Res 2005;7:R1058-79.

[30] Korkaya H, Wicha MS. HER-2, notch, and breast cancer stem cells: targeting an axis of evil. Clin Cancer Res 2009;15:1845-7.

[31] Magnifico A, Albano L, Campaner S, et al. Tumor-initiating cells of HER2-positive carcinoma cell lines express the highest oncoprotein levels and are sensitive to trastuzumab. Clin Cancer Res 2009;15:2010-21.

[32] Cornfield DB, Palazzo JP, Schwartz GF, et al. The prognostic significance of multiple morphologic features and biologic markers in ductal carcinoma in situ of the breast: a study of a 
large cohort of patients treated with surgery alone. Cancer 2004;100:2317-27.

[33] Czerniecki BJ, Koski GK, Koldovsky U, et al. Targeting HER-2/neu in early breast cancer development using dendritic cells with staged interleukin-12 burst secretion. Cancer Res 2007;67:1842-52.

[34] Leonard GD, Swain SM. Ductal carcinoma in situ, complexities and challenges. J Natl Cancer Inst 2004;96:906-20.

[35] Lari SA, Kuerer HM. Biological markers in DCIS and risk of breast recurrence: a systematic review. J Cancer 2011;2:232-61.

[36] Solin LJ, Gray R, Baehner FL, et al. A multigene expression assay to predict local recurrence risk for ductal carcinoma in situ of the breast. J Natl Cancer Inst 2013;105:701-10. [37] Rakovitch E, Nofech-Mozes S, Hanna W, et al. A population-based validation study of the DCIS score predicting recurrence risk in individuals treated by breast-conserving surgery alone. Breast Cancer Res Treat 2015;152:389-98. doi: 10.1007/s10549-015-3464-6. Epub 2015 Jun 29.

[38] NCCN Clinical Practice Guidelines in Oncology. Breast Cancer. V.2.2008. Fort Washington, PA: National Comprehensive Cancer Network, Inc; Practice Guidelines in Oncology v.2.2008. Available at: www.nccn.org/professionals/physician_gls/PDF/breast.pdf. Accessed September 24, 2014.

[39] Harris L, Fritsche H, Mennel R, et al. American Society of Clinical Oncology 2007 update of recommendations for the use of tumor markers in breast cancer. J Clin Oncol 2007;25:5287312.

[40] Pandey PR, Saidou J, Watabe K. Role of myoepithelial cells in breast tumor progression. Front Biosci 2010;15:226-36.

[41] Adriance MC, Inman JL, Petersen OW, Bissell MJ. Myoepithelial cells: good fences make good neighbors. Breast Cancer Res 2005;7:190-7.

[42] Hilson JB, Schnitt SJ, Collins LC. Phenotypic alterations in ductal carcinoma in situ- 
associated myoepithelial cells. Am J Surg Pathol 2009;33:227-32.

[43] Polyak K, Hu M. Do myoepithelial cells hold the key for breast tumor progression? J Mammary Gland Biol Neoplasia 2005;10:231-47.

[44] Gudjonsson T, Adriance MC, Sternlicht MD, Petersen OW, Bissell MJ. Myoepithelial cells: their origin and function in breast morphogenesis and neoplasia. J Mammary Gland Biol Neoplasia 2005;10:261-72.

[45] Chakrabarti A, Verbridge S, Strook AD, Fischbach C, Varner JD. Multiscale models of breast cancer progression. Ann Biomed Eng 2012;40(11): 10.1007/s10439-012-0655-8. Published online 2012 September 25.

[46] Ma XJ, Dahiya S, Richardson E, Erlander M, Sgroi DC. Gene expression profiling of the tumor microenvironment during breast cancer progression. Breast Cancer Res 2009;11:R7. [47] Schedin P, Borges V. Breaking down barriers: the importance of the stromal microenvironment in acquiring invasiveness in young women's breast cancer. Breast Cancer Res 2009;11:102. doi: 10.1186/bcr2235. Epub 2009 Mar 26.

[48] Allinen M, Beroukhim R, Cai L, et al. Molecular characterization of the tumor microenvironment in breast cancer. Cancer Cell 2004;6:17-32.

[49] Qiu, W, Hu, M, Sridhar, A, et al. No evidence of clonal somatic genetic alterations in cancer-associated fibroblasts from human breast and ovarian carcinomas. Nat Genet 2008;40:650-5.

[50] Vargas AC, McCart Reed AE, Waddell N, et al. Gene expression profiling of tumour epithelial and stromal compartments during breast cancer progression. Breast Cancer Res Treat 2012;135:153-65.

[51] Muggerud AA, Hallett $M$, Johnsen $H$, et al. Molecular diversity in ductal carcinoma in situ (DCIS) and early invasive breast cancer. Mol Oncol 2010;4:357-68.

[52] Sharma M, Beck AH, Webster JA, et al. Analysis of stromal signatures in the tumor microenvironment of ductal carcinoma in situ. Breast Cancer Res Treat. 2010;123:397-404. 
[53] Hu M, Yao J, Cai L, et al. Distinct epigenetic changes in the stromal cells of breast cancers. Nat Genet 2005;37:899-905.

[54] Fleischer T, Frigessi A, Johnson KC, et al. Genome-wide DNA methylation profiles in progression to in situ and invasive carcinoma of the breast with impact on gene transcription and prognosis. Genome Biol 2014;15:435.

[55] Van Zee KJ, Calvano JE, Bisogna M. Hypomethylation and increased gene expression of p16INK4a in primary and metastatic breast carcinoma as compared to normal breast tissue. Oncogene 1998;16:2723-7.

[56] Kristensen LS, Hansen LL. PCR-based methods for detecting single-locus DNA methylation biomarkers in cancer diagnostics, prognostics, and response to treatment. Clin Chem 2009;55:1471-83.

[57] Pang JM, Dobrovic A, Fox SB. DNA methylation in ductal carcinoma in situ of the breast. Breast Cancer Res 2013;15:206.

[58] Muggerud AA, Rønneberg JA, Wärnberg F, et al. Frequent aberrant DNA methylation of ABCB1, FOXC1, PPP2R2B and PTEN in ductal carcinoma in situ and early invasive breast cancer. Breast Cancer Res 2010;12:R3.

[59] Park SY, Kwon HJ, Lee HE, et al. Promoter CpG island hypermethylation during breast cancer progression. Virchows Arch 2011;458:73-84.

[60] Lee JS. GSTP1 promoter hypermethylation is an early event in breast carcinogenesis. Virchows Arch 2007;450:637-42.

[61] Moelans $\mathrm{CB}$, Verschuur-Maes $\mathrm{AH}$, van Diest PJ. Frequent promoter hypermethylation of BRCA2, CDH13, MSH6, PAX5, PAX6 and WT1 in ductal carcinoma in situ and invasive breast cancer. J Pathol 2011;225:222-31.

[62] Graff JR, Gabrielson E, Fujii H, Baylin SB, Herman JG. Methylation patterns of the Ecadherin 5' CpG island are unstable and reflect the dynamic, heterogeneous loss of E-cadherin expression during metastatic progression. J Biol Chem 2000;275:2727-32. 
[63] Alvarez C, Tapia T, Cornejo V, et al. Silencing of tumor suppressor genes RASSF1A, SLIT2, and WIF1 by promoter hypermethylation in hereditary breast cancer. Mol Carcinog 2013;52:475-87.

[64] Fackler MJ, McVeigh M, Evron E, et al. DNA methylation of RASSF1A, HIN-1, RAR-beta, Cyclin D2 and Twist in in situ and invasive lobular breast carcinoma. Int J Cancer 2003;107:9705.

[65] Douglas DB, Akiyama Y, Carraway H, et al. Hypermethylation of a small CpGuanine-rich region correlates with loss of activator protein-2alpha expression during progression of breast cancer. Cancer Res 2004;64:1611-20.

[66] Hoque MO, Prencipe M, Poeta ML, et al. Changes in $\mathrm{CpG}$ islands promoter methylation patterns during ductal breast carcinoma progression. Cancer Epidemiol Biomarkers Prev 2009;18:2694-700.

[67] Verschuur-Maes AH, de Bruin PC, van Diest PJ. Epigenetic progression of columnar cell lesions of the breast to invasive breast cancer. Breast Cancer Res Treat 2012;136:705-15. [68] Faryna M, Konermann C, Aulmann S, et al. Genome-wide methylation screen in low-grade breast cancer identifies novel epigenetically altered genes as potential biomarkers for tumor diagnosis. FASEB J 2012;26:4937-50.

[69] Tommasi S, Karm DL, Wu X, Yen Y, Pfeifer GP. Methylation of homeobox genes is a frequent and early epigenetic event in breast cancer. Breast Cancer Res 2009;11:R14. [70] van Hoesel $A Q$, Sato $Y$, Elashoff DA, et al. Assessment of DNA methylation status in early stages of breast cancer development. Br J Cancer 2013;108:2033-8.

[71] Bartel DP. MicroRNAs: target recognition and regulatory functions. Cell 2009;136:215-33.

[72] Croce CM. Oncogenes and cancer. N Engl J Med 2008;358:502-11.

[73] Guo H, Ingolia NT, Weissman JS, Bartel DP. Mammalian microRNAs predominantly act to decrease target mRNA levels. Nature 2010;466:835-40.

[74] Krützfeldt J, Stoffel M. MicroRNAs: a new class of regulatory genes affecting metabolism. 
Cell Metab 2006;4:9-12.

[75] Nimmo RA, Slack FJ. An elegant miRror: microRNAs in stem cells, developmental timing and cancer. Chromosoma 2009;118:405-18.

[76] Cheng AM, Byrom MW, Shelton J, Ford LP. Antisense inhibition of human miRNAs and indications for an involvement of miRNA in cell growth and apoptosis. Nucleic Acids Res $2005 ; 33: 1290-7$.

[77] Taft RJ, Pang KC, Mercer TR, Dinger M, Mattick JS. Non-coding RNAs: regulators of disease. J Pathol 2010;220:126-39.

[78] Lu J, Getz G, Miska E, et al. MicroRNA expression profiles classify human cancers. Nature 2005;435:834-8.

[79] Friedman RC, Farh KK, Burge CB, Bartel DP. Most mammalian mRNAs are conserved targets of microRNAs. Genome Res 2009;19:92-105.

[80] Griffiths-Jones S, Grocock RJ, van Dongen S, Bateman A, Enright AJ. miRBase: microRNA sequences, targets and gene nomenclature. Nucleic Acids Res 2006;34:D140-4.

[81] Lim LP, Lau NC, Garrett-Engele P, et al. Microarray analysis shows that some microRNAs downregulate large numbers of target mRNAs. Nature 2005;433:769-73.

[82] Mattie MD, Benz CC, Bowers J, et al. Optimized high-throughput microRNA expression profiling provides novel biomarker assessment of clinical prostate and breast cancer biopsies. Mol Cancer 2006;5:24.

[83] Blenkiron C, Goldstein LD, Thorne NP, et al. MicroRNA expression profiling of human breast cancer identifies new markers of tumor subtype. Genome Biol 2007;8:R214. [84] Iorio MV, Ferracin M, Liu CG, et al. MicroRNA gene expression deregulation in human breast cancer. Cancer Res 2005;65:7065-70.

[85] Hannafon BN, Sebastiani P, de las Morenas A, Lu J, Rosenberg CL. Expression of microRNA and their gene targets are dysregulated in preinvasive breast cancer. Breast Cancer Res 2011;13:R24. 
[86] Farazi TA, Horlings HM, Ten Hoeve JJ, et al. MicroRNA sequence and expression analysis in breast tumors by deep sequencing. Cancer Res 2011;71:4443-53.

[87] Volinia S, Galasso M, Sana ME, et al. Breast cancer signatures for invasiveness and prognosis defined by deep sequencing of microRNA. Proc Natl Acad Sci USA 2012;109:3024-9. [88] Reis-Filho JS. Next-generation sequencing. Breast Cancer Res 2009;11:S12.

[89] Desmedt C, Voet T, Sotiriou C, Campbell PJ. Next-generation sequencing in breast cancer: first take home messages. Curr Opin Oncol 2012;24:597-604.

[90] Vera JC, Wheat CW, Fescemyer HW, et al. Rapid transcriptome characterization for a nonmodel organism using 454 pyrosequencing. Mol Ecol 2008;17:1636-47.

[91] Emrich SJ, Barbazuk WB, Li L, Schnable PS. Gene discovery and annotation using LCM454 transcriptome sequencing. Genome Res 2007;17:69-73.

[92] Kaur H, Mao S, Li Q, et al. RNA-Seq of human breast ductal carcinoma in situ models reveals aldehyde dehydrogenase isoform 5A1 as a novel potential target. PLoS One 2012;7:e50249.

[93] Banerji S, Cibulskis K, Rangel-Escareno C, et al. Sequence analysis of mutations and translocations across breast cancer subtypes. Nature 2012;486:405-9.

Figure captions:

Figure 1: The progression to IDC.

The progression from normal breast epithelium to DCIS and IDC is characterized by alterations in the epithelial cells, myoepithelial cells, stromal cells and vascular supply. In the transition from normal epithelium to DCIS, epithelial cells undergo changes in miRNA expression and the methylation patterns of various genes; they do not demonstrate significant qualitative gene expression changes in the transition from in situ to invasive carcinoma. Myoepithelial cells undergo dramatic downregulation of many genes, causing them to lose their normal tumor suppressive abilities and allowing the epithelial cells to invade beyond the basement membrane. 
Loss of the myoepithelial cell layer is the hallmark of IDC. Angiogenesis, stimulated by local tissue hypoxia and factors elaborated by tumor-associated fibroblasts and macrophages, supports the growth of the expanding tumor.

Figure 2: Heterogeneity of myoepithelial marker expression.

Heterogeneity of expression of myoepithelial markers as detected by double immunohistochemical staining for p63 and calponin, illustrating decreased calponin staining in intermediate grade carcinoma $(A)$; strong calponin staining in apocrine DCIS (B); marked decreased in both markers in high grade DCIS with early invasion $(\mathrm{C})$, and heterogeneity in staining with decreased staining in both markers in one duct with high grade DCIS (D). (Immunohistochemistry, x400) 